\title{
Biocompatible medical implant materials with binding sites for a biodegradable drug-delivery system
}

This article was published in the following Dove Press journal:

Nanotechnology, Science and Applications

30 September 2011

Number of times this article has been viewed

\section{Haifa Al-Dubai' \\ Gisela Pittner' \\ Fritz Pittner' \\ Franz Gabor ${ }^{2}$}

'Max F Perutz Laboratories, Department of Biochemistry, University of Vienna, Vienna, Austria; ${ }^{2}$ Department of Pharmaceutical Technology and Biopharmaceutics, Faculty of Life Sciences, University of Vienna, Vienna, Austria
Correspondence: Haifa Al-Dubai

Max F Perutz Laboratories,

Department of Biochemistry,

University of Vienna,Vienna, Austria

$\mathrm{Tel}+43699$ I 3244155

Fax +43 I 42779528

Email haifa.al-dubai@univie.ac.at
Abstract: Feasibility studies have been carried out for development of a biocompatible coating of medical implant materials allowing the binding of biodegradable drug-delivery systems in a way that their reloading might be possible. These novel coatings, able to bind biodegradable nanoparticles, may serve in the long run as drug carriers to mediate local pharmacological activity. After biodegradation of the nanoparticles, the binding sites could be reloaded with fresh drug-delivering particles. As a suitable receptor system for the nanoparticles, antibodies are anchored. The design of the receptor is of great importance as any bio- or chemorecognitive interaction with other components circulating in the blood has to be avoided. Furthermore, the binding between receptor and the particles has to be strong enough to keep them tightly bound during their lifetime, but on the other hand allow reloading after final degradation of the particles. The nanoparticles suggested as a drug-delivery system for medical implants can be loaded with different pharmaceuticals such as antibiotics, growth factors, or immunosuppressives. This concept may enable the changing of medication, even after implantation of the medical device, if afforded by patients' needs.

Keywords: antibody immobilization, biocompatible coating, chitosan nanoparticles, drug targeting, medical device

\section{Introduction}

Drug-delivering implants such as drug-eluting stents (DESs) play an important role in the treatment of cardiovascular diseases. These stents have been introduced as a new and promising technique to reduce restenosis after stent implantation. Several substances with immunosuppressive, antiproliferative, and cytostatic activities have been tested as active stent coatings and have been shown to reduce restenosis in humans. ${ }^{1,2}$ Despite these advantages, restenosis rates still remain substantial in high-risk patients, such as patients with long lesions and complex lesion morphologies in bifurcations. The DESs currently in use contain only a certain amount of drug, and restenosis may take place if this reservoir is used up. These limitations might be overcome through reloading the stent (or other implant material) with drugs, as proposed in the setup outlined in this paper.

Furthermore, the here presented biocompatible coating of implant materials with binding sites for drug-delivering nanoparticles (NPs) might also be useful for other medical implants as specially designed joints to improve the healing process.

The general design approach developed in this overall concept is: medical implant materials are coated with a covalently bound biocompatible layer. This forms a passive protection layer preventing rejection of the implant material. Additionally, antibodies 
(Abs) against chitosan (CS)-NPs were anchored in this protective layer acting as specific biorecognitive sites. These were subsequently used to load biodegradable, drugcontaining CS-NPs onto the implant. After implantation of the medical device into the patient, such a structure might serve as a reloadable drug-delivery system. Biodegradable NPs containing pharmaceutical drugs can be injected, and while circulating in the bloodstream can bind to these specific binding sites. The particles are then slowly degraded by enzymes in the surrounding body fluids, thus gradually releasing the drugs. At the end of this process, after complete degradation of the NPs, the biorecognitive sites should be free again to be reloaded with new drug-containing particles (Figure 1).

The possibility of reloading might allow not only patient-adequate dosage of drugs but also change of medication after implantation, using different drugs at different stages of the healing process. In case of bone implants, NPs can be loaded with growth factors that promote replication, differentiation, protein synthesis, and/or migration of respective cell types. The successful development of this technology will provide a better means for controlled drug release from the implant due to the site-directed binding of the drug-carrier NPs to the implant surfaces. After implantation, the patient should benefit because of a more efficient and directed drug release at the target site, thus improving the healing process.
The biocompatible coating in this study is based on polyethylene glycol (PEG) derivatives which are covalently bound to the backbone carrier, serving as a passive coating. PEG is employed extensively in pharmaceutical and biomedical applications. It can be chemically modified for attachment to other molecules and surfaces. ${ }^{3}$

The drug-delivery system in this study is based on CS-NPs. The low toxicity, ${ }^{4}$ excellent capacity for the entrapment of proteins, and the chemical properties of CS make it a suitable candidate for use in gene- and drug-delivery systems. ${ }^{5-7} \mathrm{CS}$ has been a focus of increasing attention in the design and engineering of novel nanoparticulate drug-delivery systems, due to their desirable properties such as biocompatibility, biodegradability, bio- and mucoadhesivity, as well as hydrophilic character that facilitate the administration and increase the bioavailability of poorly absorbable drugs across various epithelial barriers, such as corneal, nasal, and intestinal mucosa. ${ }^{8}$ An additional advantage of CS-NPs is their production under aqueous and fairly mild conditions, thus being especially suitable to preserve the bioactive conformation of delicate macromolecules (eg, proteins, hormones, antigens, pDNA [plasmid deoxyribonucleic acid], siRNA [small interfering ribonucleic acid], growth factors, heparin, etc) that otherwise would be prone to enzymatic degradation and hydrolysis., ${ }^{9,10}$ The physicochemical, biomedical, and pharmaceutical properties of CS have already been described in detail in several articles. ${ }^{11-17}$ Drug-delivery systems including

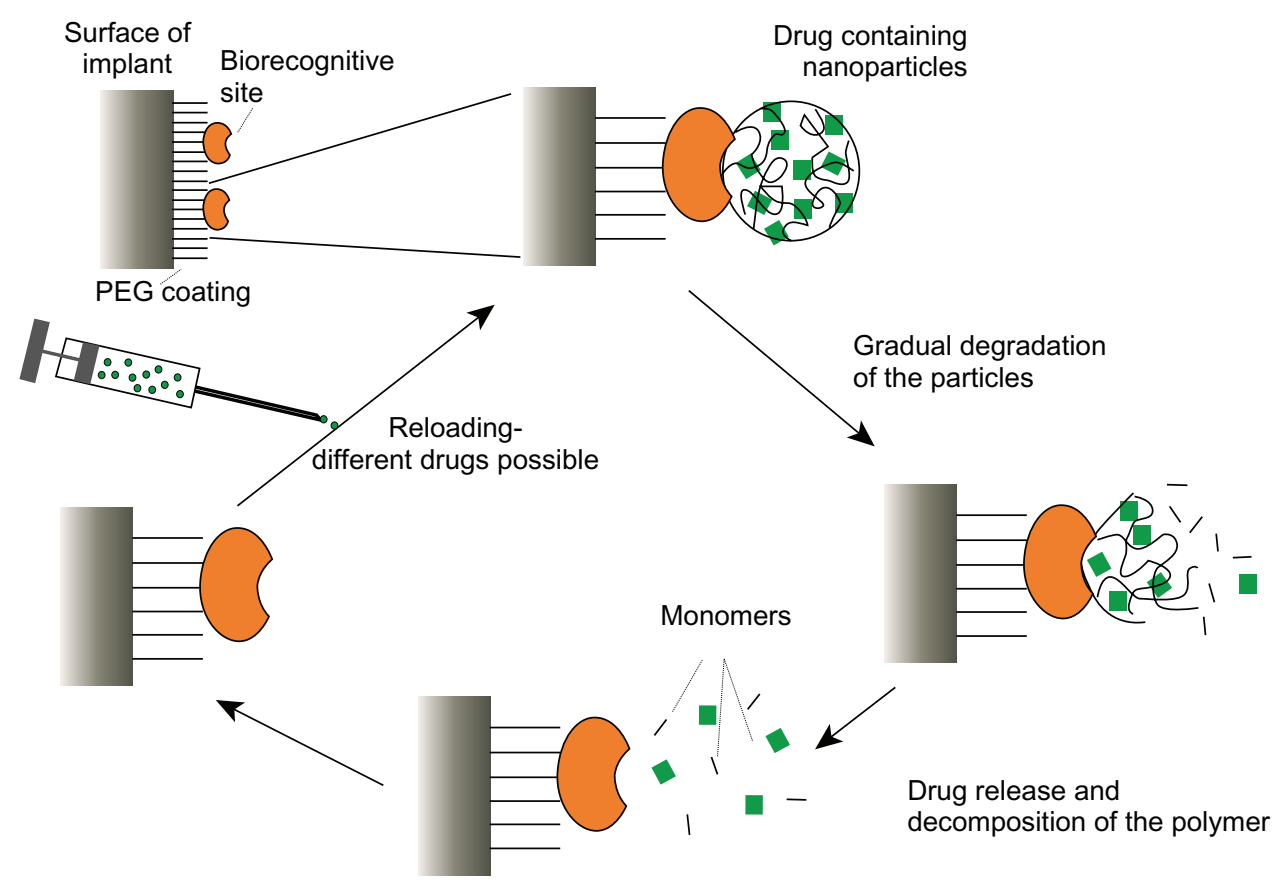

Figure I Principle of nanoparticle binding, drug release, and reloading. Abbreviation: PEG, polyethylene glycol. 
CS-beta-cyclodextrin NPs and nanocore-coated type capsules was the focus of Alonso et al's study. ${ }^{8} \mathrm{CS}$ is a linear amino polysaccharide containing approximately 20\% 1,4-linked N-acetyl-D-glucosamine (GlcNAc) and approximately $80 \%$ $\beta 1,4$-linked D-glucosamine ( $\mathrm{GlcN})$ and is produced by partial deacetylation of chitin in hot alkali. CS is degradable by lysozyme circulating also in the bloodstream, thus making it a welcome material for the production of drug-containing NPs being slowly degraded and thus releasing their content. ${ }^{17}$

The interaction between the CS-fluorescence-labeled NPs (containing boron-dipyrromethene [BODIPY] 493/503 as a fluorescent tag) and their receptor (Abs) immobilized onto the carrier surface is shown in this study using fluorescence microscopy. As this analytical method affords very smooth and nonquenching surfaces, glass slides were used as a support material for the coating. The here presented setup for coating was also successfully performed for different medically used implant materials as referred to in the section "Results and discussion."

\section{Materials and methods Organic silanization of inorganic carriers}

For the reasons already mentioned in the introduction, silicate glass slides (Ø12 mm, Assistant, Vienna, Austria) were used as inorganic carriers. These slides were etched with $1 \mathrm{M} \mathrm{NaOH}$ for 30 minutes, then washed several times with double-distilled water $\left(\mathrm{ddH}_{2} \mathrm{O}\right)$, and finally soaked for 2 days in $\mathrm{ddH}_{2} \mathrm{O}$ under gentle shaking. Replacement of $\mathrm{ddH}_{2} \mathrm{O}$ several times to enhance hydroxyl group formation is highly recommended. The surface of the carriers was then modified by amino-silanization with 3-aminopropyl triethoxysilane (Sigma-Aldrich, Vienna, Austria) ${ }^{18}$ in order to immobilize either the bifunctional bis-amino-PEG $\left(\mathrm{NH}_{2}-\mathrm{PEG}-\mathrm{NH}_{2}\right)$ or monofunctional mercapto-PEG (SH-PEG). The used carriers were covered with freshly prepared 5\% (v/v) (3-aminopropyl) triethoxysilane solution in $95 \% \mathrm{EtOH}$ and reacted for 1 hour under gentle shaking at room temperature, then washed three times for 5 minutes with 95\% (v/v) EtOH under gentle shaking and cured at $110^{\circ} \mathrm{C}$ overnight.

\section{Binding of succinic anhydride to the amino-silanized surface}

Succinic anhydride is applied to introduce carboxylate groups onto the surface of amino-silanized material: $1 \mathrm{~g}$ of succinic anhydride (Merck, Vienna, Austria) was suspended in $25 \mathrm{~mL}$ phosphate buffered saline (PBS) buffer $\mathrm{pH}$ 8.4. The $\mathrm{pH}$ was monitored and adjusted to 6 with $1 \mathrm{M} \mathrm{NaOH}$ to prevent severe acidification of the reaction solution, which might damage the molecule to be coupled in the further step. This solution was added to the amino-silanized carrier and reacted at room temperature overnight under shaking with an orbital shaker to assure complete blocking of all amino groups (controlling the $\mathrm{pH}$ over the first few hours of the reaction). The carboxylated carrier was washed three times for 5 minutes with buffer, followed by three times with $\mathrm{ddH}_{2} \mathrm{O}$.

\section{EDC combined with sulfo-NHS}

A solution of 0.1 mM EDC (1-ethyl-3-(3-dimethylaminopropyl) carbodiimide, Sigma-Aldrich) and sulfo-NHS (N-hydroxysulfosuccinimide, Pierce, Lausanne, Switzerland) in a final

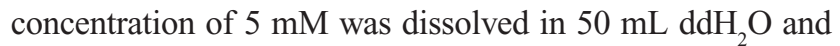
the $\mathrm{pH}$ adjusted to 10 with $1 \mathrm{M} \mathrm{NaOH} .{ }^{18,19}$ The carboxylated carrier prepared with succinic anhydride was incubated in this solution for about 1-2 hours (maximum) at room temperature under gentle shaking. The activated carrier with EDC/sulfoNHS was washed several times with $\mathrm{ddH}_{2} \mathrm{O}$.

\section{Coupling of $p$-chloro-anil as a linker}

As $p$-chloro-anil is sensitive to light, all reaction steps have to be carried out under light protection ${ }^{18}$ until $\mathrm{NH}_{2}$-PEG$\mathrm{NH}_{2}$ or $\mathrm{Ab}$ is immobilized. The amino-silanized carrier was washed two times with toluene (JT Baker, Vienna, Austria) for 5 minutes under shaking then incubated with a $1 \%(\mathrm{w} / \mathrm{v})$ solution of $p$-chloro-anil (Fluka, Vienna, Austria) in toluene for 1 hour at room temperature under gentle shaking. The activated carrier $p$-chloro-anil was washed two times with toluene, acetone (JT Baker) and $\mathrm{dd}_{2} \mathrm{O}$ respectively.

\section{Amino-PEGylation}

1 g O,O'-bis (3-aminopropyl) PEG $\left(\mathrm{NH}_{2}-\mathrm{PEG}-\mathrm{NH}_{2}\right.$, molecular weight [MW] 1500, Fluka) was dissolved in $50 \mathrm{~mL}$ of $0.1 \mathrm{M}$ citric acid-sodium citrate buffer $\mathrm{pH} 5$ or $50 \mathrm{~mL}$ of $0.1 \mathrm{M}$ carbonate-bicarbonate buffer $\mathrm{pH} 10$ respectively. The carrier, activated with either EDC/sulfo-NHS or $p$-chloroanil, was incubated in the above prepared solutions separately at least overnight. Each amino-PEGylated carrier was washed three times for 5 minutes under shaking with the buffer to remove unreacted PEG and then washed several times with $\mathrm{ddH}_{2} \mathrm{O}$. In case of $p$-chloro-anil, crosslinker free-binding sites were blocked using ethanolamine $(1: 10, \mathrm{v} / \mathrm{v})$ in $\mathrm{ddH}_{2} \mathrm{O}$ for 30 minutes on an orbital shaker.

\section{SH-PEGylation}

SH-PEG (MW 5000) was provided from SigmaAldrich. Amino-silanized carriers already activated with 
sulfo-SMCC (sulfosuccinimidyl-4-(N-maleimidomethyl)cyclohexane-1-carboxylate) and then coupled with $\mathrm{CS}-\mathrm{Ab}$ (see "Immobilization of Abs onto the carrier" below) were incubated in $500 \mu \mathrm{L}$ of a solution of $121 \mathrm{mg} / \mathrm{mL}$ SH-PEG (dissolved in $50 \mathrm{mM}$ sodium phosphate buffer $\mathrm{pH}$ 7.3) per carrier (Ø12 mm silicate glass cover slides) under argon in a humid chamber overnight at $4^{\circ} \mathrm{C}$ under shaking. The carriers were washed three times for 5 minutes with Tween ${ }^{\circledR} 20$ washing solution (washing solution contains $0.5 \%$ (v/v) Tween 20 (Sigma-Aldrich) and 0.1 M phosphate buffer, $0.15 \mathrm{M}$ $\mathrm{NaCl} \mathrm{pH} 7.3$ ) on an orbital shaker. The washed carriers were blocked in 2-mercaptoethanol ( $\beta$-ME, Fluka) blocking solution (35 mM $\beta$-ME in 0.1 M sodium phosphate buffer $\mathrm{pH} 7.3$ ) for 30 minutes under shaking. The blocked carriers were washed as before and incubated with fluorescent CS-NPs.

\section{Immobilization of Abs onto the carrier}

The amino-PEGylated or amino-silanized carriers ( $4 \times 012 \mathrm{~mm}$ silicate glass cover slides) were washed with PBS buffer (0.1 M PB with 0.15 M NaCl, pH 7.2).

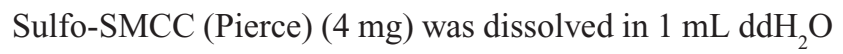
and then filled to $11 \mathrm{~mL}$ with the abovementioned $0.1 \mathrm{M}$ PBS buffer. The carriers were incubated for 30 minutes in this solution under gentle shaking. The carriers were washed 3 times with buffer for 5 minutes under gentle shaking. The used Abs for capture of the CS-NPs were a mouse monoclonal anti-ß-O-linked acetylglucosamine (O-GlcNAc) IgM isotype (Sigma-Aldrich). Throughout this paper, it is referred to as CS-Ab. Uncleaved CS-Ab $(2 \mathrm{mg} / \mathrm{mL}$ diluted 1:55 in $50 \mathrm{mM} \mathrm{PB} \mathrm{pH} \mathrm{7.5)} \mathrm{was} \mathrm{pipetted} \mathrm{onto} \mathrm{the} \mathrm{carrier}$ activated with either sulfo-SMCC or $p$-chloro-anil and incubated for 1 hour at $37^{\circ} \mathrm{C}$. The carrier was washed three times for 5 minutes with Tween 20 washing solution under gentle shaking. Silicate glass carriers with Abs immobilized by means of sulfo-SMCC crosslinker were blocked with $35 \mathrm{mM}$ B-ME in $0.1 \mathrm{M}$ sodium phosphate $\mathrm{pH} 7.3$ for 30 minutes under shaking. Silicate glass carriers containing Abs immobilized via $p$-chloro-anil crosslinker were blocked using ethanolamine $(1: 10, \mathrm{v} / \mathrm{v})$ in $\mathrm{dd}_{2} \mathrm{O}$ for 30 minutes on an orbital shaker. Silicate glass carriers for negative control were amino-silanized, activated with $p$-chloro-anil and activated with sulfo-SMCC omitting immobilization of CS-Abs. After the blocking step, the silicate glass surfaces were washed three times for 5 minutes under shaking in Tween 20 washing solution and subsequently rinsed with $\mathrm{ddH}_{2} \mathrm{O}$. Fluorescent CS-NPs (see "Preparation of CS-NPs" below) were diluted 1:10 with $\mathrm{ddH}_{2} \mathrm{O}$. Diluted fluorescent CS-NPs $(100 \mu \mathrm{L})$ were pipetted onto each washed carrier. The carriers were then incubated overnight at $4^{\circ} \mathrm{C}$ in a humid chamber. The carriers were washed three times for 5 minutes with Tween 20 washing solution under shaking. Binding of fluorescent CS-NPs to the silicate glass carriers was visualized by fluorescent microscopy (Olympus BX41, Olympus, Tokyo, Japan) using excitation/emission wavelengths of 493/503 nm for BODIPY 493/503. Images were taken in ColorView software (Soft Imaging System) at 40× magnification and edited in $\mathrm{Cell}^{\wedge} \mathrm{D}$ life science documentation software (Olympus Soft Imaging Solutions, Münster, Germany).

\section{Preparation of CS-NPs}

CS-NPs were prepared according to Calvo et al. ${ }^{5}$ A CS solution $(2 \mathrm{mg} / \mathrm{mL})$ in $5 \%$ stoichiometric excess of acetic acid (HAc) was prepared. In the present study, theoretically $0.063 \%$ HAc was needed for CS (Sigma-Aldrich) with a deacetylation degree of $75 \%-85 \%$ and a $\mathrm{MW}$ between 50,000 and 190,000 . The resulting $\mathrm{pH}$ of the solution was adjusted to 4.3 with 5\% HAc to obtain a clear solution of CS. A sodium tripolyphosphate (TPP) (Sigma-Aldrich) stock solution in a concentration of $3 \mathrm{mg} / \mathrm{mL}$ was prepared in $\mathrm{ddH}_{2} \mathrm{O}$. A volume of $5 \mathrm{~mL}$ of the following dilutions of the TPP stock was prepared (TPP stock:water): (1) stock solution, (2) $2: 1$, (3) $1: 1$, and (4) 1:2. An aliquot of $3 \mathrm{~mL} \mathrm{CS}$ solution was pipetted into a silicate glass test tube that was placed upright in a $50 \mathrm{~mL}$ Erlenmeyer flask. TPP solution $(1 \mathrm{~mL}$, prepared as detailed before) was added under stirring into the center of the vortex formed in the CS solution in the test tube. After adding TPP, the opalescence of the solution increased with TPP concentration. After stirring for 10 minutes, the mixture was kept standing for 10 minutes. Then the solution was visually inspected for aggregates. Formation of visible particles is a sign of unwanted aggregation. The final $\mathrm{pH}$ of the NP suspension is shown in Table 1. With stock solution, precipitation was observed. Particle size was measured for the formulations that showed no aggregation or precipitation. A 1:1 dilution of TPP was found to give best results (Table 1). The mean CS-NPs size and distribution were determined by dynamic light scattering (DLS) (Zetasizer Nano ZS, Malvern

Table I Size of prepared CS-NPs

\begin{tabular}{llll}
\hline TPP-dilution $^{\mathbf{a}}$ & pH of NP suspension & $\begin{array}{l}\text { Size of NPs } \\
(\mathbf{n m})\end{array}$ & PDI \\
\hline $2: 1$ & 5.05 & 352 & 0.499 \\
$\mathrm{I}: \mathrm{I}$ & 4.97 & 253 & 0.280 \\
$\mathrm{I}: 2$ & 4.87 & 293 & 0.293 \\
\hline
\end{tabular}

Note: ${ }^{a} 3 \mathrm{mg} / \mathrm{mL}$ TPP stock: water

Abbreviations: CS, chitosan; NP, nanoparticle; PDI, polydispersity index; TPP, sodium tripolyphosphate. 
Instruments Ltd, Worcestershire, UK). All measurements were carried out in triplicate at $20^{\circ} \mathrm{C}$ after 5 minutes of equilibration time.

After formation, the NPs were recovered by centrifugation: $1 \mathrm{~mL}$ of the NP suspension was transferred into $1.5 \mathrm{~mL}$ Eppendorf vials with a $20 \mu \mathrm{L}$ glycerol bed at the bottom of the vial (glycerol is used to avoid aggregation and facilitate resuspension of the isolated NPs). The vials were centrifuged at $10,000 \times g$ for 40 minutes at $25^{\circ} \mathrm{C}$, and subsequently the supernatant was removed. A volume of $100 \mu \mathrm{L}$ of water was added and the NP-pellet resuspended with a $100 \mu \mathrm{L}$ micropipette by vigorous, repeated mixing.

The preparation of fluorescent CS-NP tagged with BODIPY 493/503 (4,4-difluoro-1,3,5,7,8-pentamethyl-4bora-3a,4a-diaza-s-indacene, Invitrogen, Carlsbad, CA) was carried out as described above. In this case, $100 \mu \mathrm{L}$ of BODIPY 493/503 solution (dissolved in ethylacetate at a concentration of $1 \mathrm{mg} / \mathrm{mL}$ ) was added to $3 \mathrm{~mL} \mathrm{CS} \mathrm{solu-}$ tion $(2 \mathrm{mg} / \mathrm{mL})$ by stirring vigorously at room temperature for 20 minutes. The solution was filtered through a $5 \mu \mathrm{m}$ membrane filter (sterile MILLEX ${ }^{\circledR}-S V$, Merck Millipore, Billerica, MA) and $1 \mathrm{~mL}$ of TPP solution (1:1) was added as described above. The size of NPs tagged with BODIPY $493 / 503$ was $275.1 \mathrm{~nm}$, and their polydispersity index (PDI) was 0.239. Labeled NPs were centrifuged, resuspended in $100 \mu \mathrm{L} \mathrm{ddH_{2 }} \mathrm{O}$ as already described, and used for detection of immobilized $\mathrm{CS}-\mathrm{Ab}$ on either amino-silanized silicate glass surfaces, or amino-PEGylated or SH-PEGylated silicate glass surfaces.

\section{Results and discussion}

As examples for medical implant materials, stainless steel L316 as well as zirconia (ceramics) were used. The successful setup procedure of the biocompatible coating was already shown for these materials as well as for silicate glass. ${ }^{20}$ Because of the uneven surface of zirconia and quenching of optical signals in case of metals silicate glass coated under the same conditions was used as a model substance when using fluorescence labeling as an analytical tool.

For immobilization of the biocompatible coating using either the bifunctional $\mathrm{NH}_{2}-\mathrm{PEG}_{-} \mathrm{NH}_{2}$ (amino-PEGylation) or monofunctional SH-PEG (SH-PEGylation) the surface of used carriers needs to be previously activated via chemical etching followed by amino-silanization. ${ }^{18} \mathrm{Bi}$-functional PEGs or SH-PEG were bound covalently to the previously aminosilanized carrier surface to enhance biocompatibility. The choice of PEG-coupling procedure depends on the functional groups of the PEG derivative. For amino-PEGylation in a first step, succinic anhydride was coupled to the aminosilane followed by EDC/sulfo-NHS activation. ${ }^{18,19}$ Bis-amino-PEG can also be coupled directly to the amino-silanized carrier using $p$-chloro-anil as a crosslinker (Figure 2). p-Chloro-anil linkers react with amino groups and thus can be coupled to the silane moiety under mild conditions. ${ }^{18}$ In a second reaction step, the amino groups of a polymer as, for example, $\mathrm{NH}_{2}-\mathrm{PEG}-\mathrm{NH}_{2}$ can then be coupled in para-position.

The immobilization of the biorecognitive binding site (Abs) for drug delivery (CS-NPs) onto the PEGylated or amino-silanized carrier was carried out by using either the sulfo-SMCC crosslinking method or the $p$-chloro-anil method. ${ }^{21,18}$ In the here presented system, the used CS-Ab for capture of the CS-NPs was a mouse monoclonal anti- $\beta$ O-linked acetylglucosamine (O-GlcNAc) IgM isotype that recognizes O-GlcNAc. CS-Ab molecules are immobilized onto the terminal amino groups of the amino-PEGylated carrier or directly onto an amino-silanized carrier using either the sulfoSMCC (Figure 3) or the $p$-chloro-anil crosslinking method.

The SH-PEGylation using monofunctional SH-PEG was carried out on amino-silanized slides already containing $\mathrm{CS}-\mathrm{Ab}$ molecules. The coupling of CS-Abs using sulfoSMCC crosslinker was then followed by SH-PEGylation. The free maleimide end of sulfo-SMCC not bound to CS-Abs thus couples specifically to the sulfhydryl groups of SH-PEG (Figure 3).

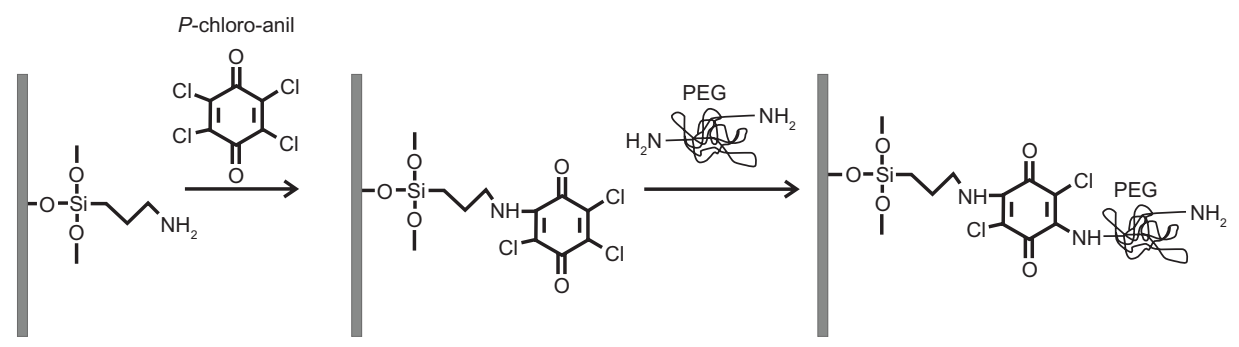

Figure 2 Use of $p$-chloro-anil as a crosslinker between the silane moiety and the amino groups of a polymer such as $\mathrm{NH}_{2}-\mathrm{PEG}-\mathrm{NH}_{2}$ or protein $\mathrm{Abs}$ (eg, CS-Abs), coupled in para-position.

Abbreviations: Abs, antibodies; CS, chitosan; PEG, polyethylene glycol. 


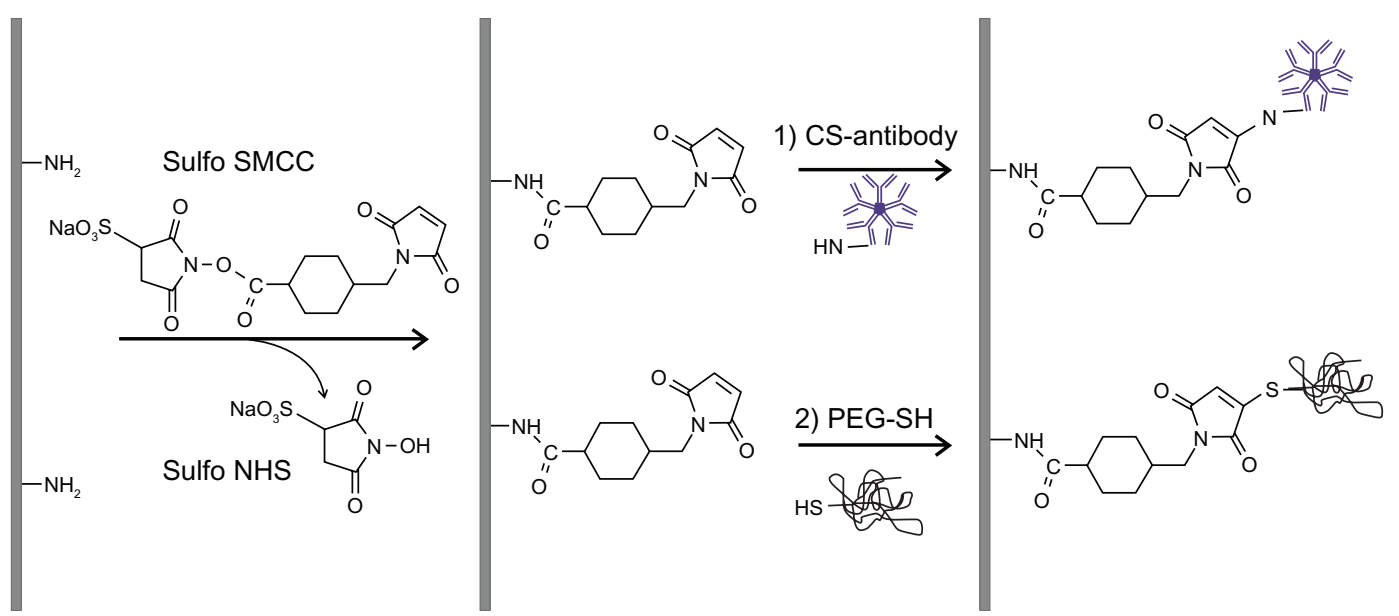

Figure 3 Binding of whole CS-antibodies onto the carrier via sulfo-SMCC, followed by reaction with PEG-SH for enhancing biocompatibility. Any unreacted sites were finally blocked with B-ME.

Abbreviations: CS, chitosan; sulfo-SMCC, sulfosuccinimidyl-4-(N-maleimidomethyl)-cyclohexane-I-carboxylate; sulfo-NHS, N-hydroxysulfosuccinimide; B-ME, $\beta$-mercaptoethanol; PEG-SH, (O-[2-(3-mercaptopropionylamino) ethyl]-O'-methyl-polyethylene glycol).

The interaction between CS-NPs and their Abs immobilized onto a carrier was demonstrated employing CS-fluorescencelabeled particles using fluorescence microscopy.

\section{Formation of CS-NPs}

The formation of CS-NPs occurs spontaneously under mild gelation conditions with no need for sonication, high temperatures, or organic solvents. It has been shown earlier that the inter- and intramolecular linkages created between the positively charged amino groups of CS and the negatively charged TPP are responsible for the initiation of the gelation process. ${ }^{5}$ The gelation conditions were optimized using different dilutions of TPP. A concentration of $1.5 \mathrm{mg}$ $\mathrm{TPP} / \mathrm{mL} \mathrm{ddH}_{2} \mathrm{O}$ was found to be the best condition for the formation NPs, with a size of $253 \mathrm{~nm}$ and a PDI of 0.280 . CS-NPs generated under these conditions were tagged with fluorescent marker molecules (BODIPY 493/503). The size of CS-NPs conjugated to BODIPY 493/503 was $275.1 \mathrm{~nm}$ and its PDI was 0.239 .

\section{Immobilization of recognitive binding sites for CS-NPs onto amino-silanized carriers}

One of the aims of this study was to develop a process that allows coating of carriers with a biocompatible layer that provides free-binding sites suitable to load drug molecules onto the implant. This was achieved by immobilizing CS-Ab molecules onto a carrier as binding sites for CS-NPs. This approach was demonstrated successfully first on aminosilanized carriers (Figure 4). CS-NPs were labeled with fluorophores and binding to their binding site (CS-Abs) immobilized on carrier using either sulfo-SMCC (Figure 4A) or $p$-chloro-anil (Figure 4C) was shown by fluorescence microscopy. In negative controls, omitting CS-Abs no fluorescent CS-NPs could be detected in sulfo-SMCC carriers (Figure 4B) and only a low background is observed with p-chloro-anil carriers (Figure 4D).

The obviously uneven distribution of biding sites on the carrier might derive from problems of etching very smooth surfaces.

\section{Organic silanization}

1: Sulfo-SMCC crosslinker
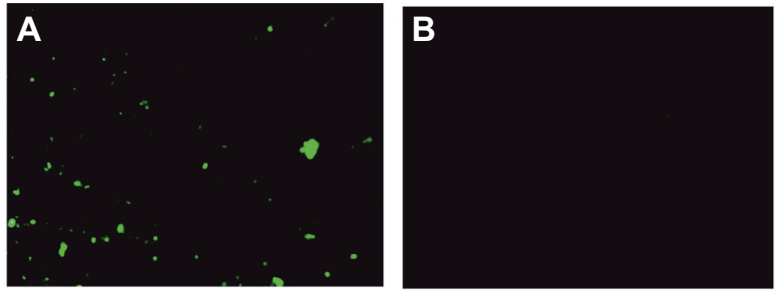

2: P-chloro-anil crosslinker
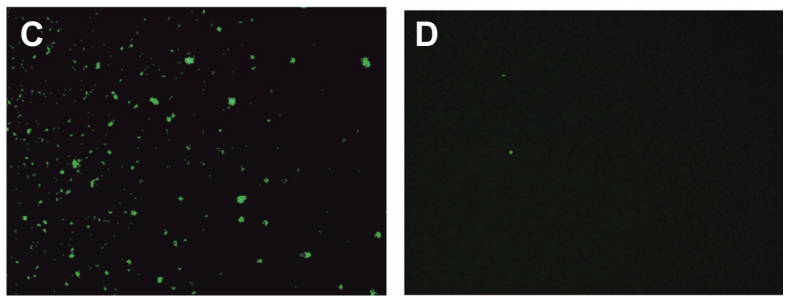

Figure 4 Binding efficiency of CS-NPs labeled with BODIPY 493/503 by immobilization of CS-Ab by means of (A) sulfo-SMCC or (C) p-chloro-anil. (B and D) Negative control (without immobilized CS-Ab).

Abbreviations: CS-NPs, chitosan-nanoparticles; BODIPY 493/503, 4,4-difluoroI,3,5,7,8-pentamethyl-4-bora-3a,4a-diaza-s-indacene; CS-Ab, chitosan-antibody; sulfo SMCC, sulfosuccinimidyl-4-( $\mathrm{N}$-maleimidomethyl)-cyclohexane-I-carboxylate. 


\section{SH-PEGylation of carriers containing CS-Abs}

The SH-PEGylation at $\mathrm{pH} 7.3$ was carried out on the aminosilanized carrier after the coupling of CS-Ab by means of a sulfo-SMCC crosslinker according to Figure 3 to enhance biocompatibility of the carrier. The interaction between immobilized CS-Ab and the labeled CS-NPS on the biocompatible surface is presented in Figure 5A, whereas no signal was observed in a negative control without immobilized $\mathrm{CS}-\mathrm{Ab}$ (Figure 5B).

\section{Immobilization of recognitive binding sites for CS-NPs onto amino-PEGylated carriers}

The amino-pEGylation of carriers was done by means of EDC/sulfo-NHS or $p$-chloro-anil at different $\mathrm{pH}$ (5 or 10). Amino-PEGylation at $\mathrm{pH} 5$ results in a higher binding affinity of CS-NPs (Figures 6A and $\mathrm{C}$ ) than at $\mathrm{pH}$ 10 (data not shown). That was consistent with the hypothesis that under acidic conditions amino groups on PEG tend to the surface of the molecule because of stronger interactions with the hydrophilic surrounding and thus become available for binding of the crosslinkers (EDC/ sulfo-NHS, Figure 6A and B; or $p$-chloro-anil, Figure 6C and D), necessary for further immobilization of CS-Abs using sulfo-SMCC.

Figure 6 demonstrates also the different crosslinking efficiency of EDC/sulfo-NHS and $p$-chloro-anil used for amino-PEGylation. Coating of $\mathrm{NH}_{2}-\mathrm{PEG}-\mathrm{NH}_{2}$ using EDC/ sulfo-NHS is more time consuming than the $p$-chloro-anil method, but $p$-chloro-anil gives some low background (Figure 6D), indicating incomplete reaction.

EDC/sulfo-NHS may be the method of choice for polymer coating of an implant surface, as the resulting amide bond linkage does not elicit any immunogenic response.

\section{Coating with SH-PEG}
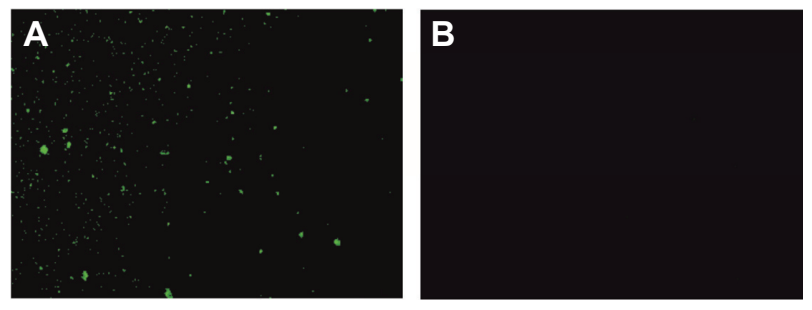

Figure 5 Fluorescence microscopy images. (A) Coated silicate glass carriers containing immobilized CS-Abs followed by SH-PEGylation. (B) No signal was observed for the negative control (omitting the immobilization of CS-Ab).

Abbreviations: CS-Ab, chitosan-antibody; SH-PEGylation, coating with PEG-SH; PEG-SH, (O-[2-(3-mercaptopropionylamino) ethyl]-O'-methyl-polyethylene glycol).
Coating with bis-amino-PEG (pH 5)

1: EDC/Sulfo-NHS crosslinker
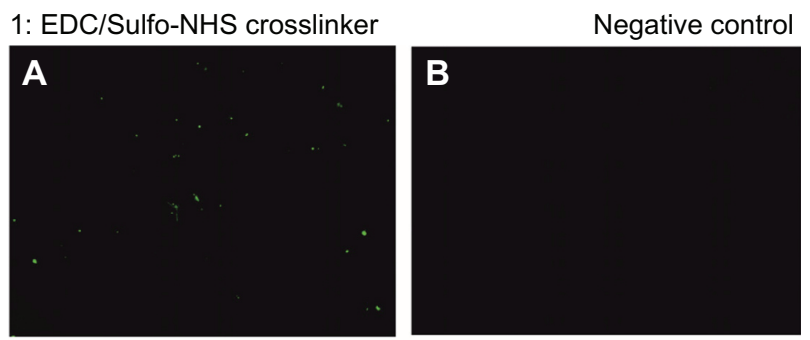

2: P-chloro-anil crosslinker
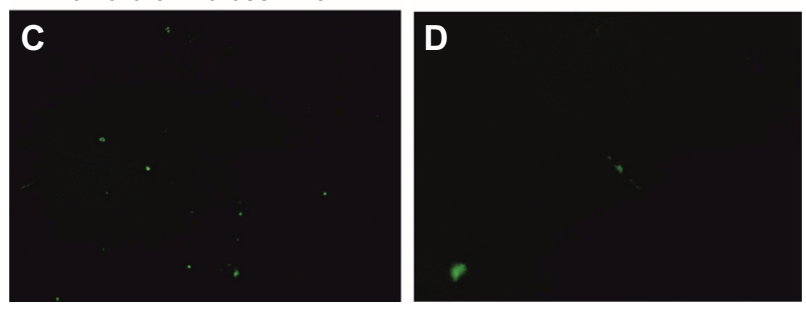

Figure 6 Fluorescence microscopy images of carriers coated with $\mathrm{NH}_{2}-\mathrm{PEG}-\mathrm{NH}_{2}$ at $\mathrm{pH}$ 5. The crosslinkers (either EDC/sulfo-NHS or p-chloro-anil) were used to immobilize $\mathrm{NH}_{2}-\mathrm{PEG}-\mathrm{NH}_{2}$ onto organic amino-silanized carriers. The immobilization of CS-Ab was applied by means of sulfo-SMCC.

Abbreviations: $\mathrm{CS}-\mathrm{Ab}$, chitosan antibody; $\mathrm{NH}_{2}-\mathrm{PEG}-\mathrm{NH}_{2}$, bis-amino-PEG; EDC, I-ethyl-3-(3-dimethylaminopropyl) carbodiimide; sulfo-NHS, N-hydroxysulfosuccinimide; sulfo-SMCC, sulfosuccinimidyl-4-(N-maleimidomethyl)-cyclohexane-I-carboxylate.

\section{Conclusion}

Drug-eluting materials existing so far contain drug volumes sufficient only for several weeks after implantation without reloading. For development of novel implant material with reloading properties carrier backbone was covalently bound to PEG polymer. This passive coating serves as a barrier with good biocompatibility on the surface of the implant materials. The active coating is based on the effect of drugs loaded into NPs (such as CS-NPs, which can be degraded by lysozyme circulating in the bloodstream), which bind to their immobilized recognitive site (Abs) on the surface of carrier materials (implant material). The interaction of CSfluorescence-labeled NPs and their immobilized CS-Abs was successfully shown using fluorescence microscopy.

\section{Acknowledgments}

The authors thank Dr Francisco M Goycoolea Valencia (University Santiago de Compostela, Spain) for his helpful advice concerning preparation of CS-NPs, Prof Irene Lichtscheidl (Institute of Cell Imaging and Ultrastructure Research, Vienna, Austria) for using microscopy in her lab, and the Department of Biochemistry, University of Vienna (Dr Bohrgasse 9, A-1030 Vienna, Austria) for financial support.

\section{Disclosure}

The authors report no conflicts of interest in this work. 


\section{References}

1. Chieffo A, Colombo A. Drug-eluting stents. Minerva Cardioangiol. 2002;50(5):419-429.

2. Grube E, Sonoda S, Ikeno F, et al. Angiographic results of the first human experience with Sirolimus-eluting stents for the treatment of coronary lesions. Circulation. 2004;109:2168-2171.

3. Ratner BD, Hoffman AS, Schoen FJ, Lemons JE, editors. An introduction to materials in medicine. In: Biomaterials Science. 2nd ed. SanDiego, CA: Elsevier; 2004:1-209.

4. Aspden TJ, Illum L, Skaugrud O. The absence of chitosan toxicity when applied to nasal mucosa. Proc Intern Symp Control Rel Bioact Mater. 1995;22:550-556.

5. Calvo P, Remuñán-López C, Vila-Jato JL, Alonso MJ. Novel hydrophilic chitosan-polyethylene oxide nanoparticles as protein carriers. $J$ Appl Polym Sci. 1997;63:125-132.

6. Calvo P, Remuñán-López C, Vila-Jato J, Alonso MJ. Chitosan and chitosan/ethylene oxide-propylene oxide block copolymer nanoparticles as novel carriers for protein and vaccines. Pharm Res. 1997; 14:1431-1436.

7. De la Fuente M, Seijo B, Alonso MJ. Novel hyaluronic acid-chitosan nanoparticles for ocular gene therapy. Invest Ophthalmol Vis Sci. 2008;49:2016-2024.

8. Alonso MJ, Prego C, García-Fuentes M. Polysaccharide-based nanoparticles as carriers for drug and vaccine delivery. In: Domb J, Tabata Y, Ravi Kumar MNV, Farber S, editors. Nanoparticles for Pharmaceutical Application. Valencia, CA: American Scientific Publishers; 2007:135-150.

9. Janes KA, Calvo P,Alonso MJ. Polysaccharide colloidal particles as delivery systems for macromolecules. Adv Drug Del Rev. 2001;47:83-97.

10. Csaba N, Garcia-Fuentes M, Alonso MJ. The performance of nanocarriers for transmucosal drug delivery. Expert Opin Drug Deliv. $2006 ; 3: 463-478$.
11. Skaugrud O, Hagen A, Borgersen B, Dornish M. Biomedical and pharmaceutical applications of alginate and chitosan. Biotechnol Genet Eng Rev. 1999;16:23-40.

12. Agnihotri A, Mallikarjuna NN, Aminabhavi TM. Recent advances on chitosan-based micro- and nanoparticles in drug delivery. $J$ Control Rel. 2004;100:5-28.

13. Ravi Kumar MNV, Muzzarelli RAA, Muzzarelli C, Sashiwa H, Domb AJ. Chitosan chemistry and pharmaceutical perspectives. Chem Rev. 2004;104:6017-6084.

14. George M, Abraham TE. Polyionic hydrocolloids for the intestinal delivery of protein drugs: alginate and chitosan - a review. J Control Rel. 2006;114:1-14.

15. Rinaudeo M. Chitin and chitosan: properties and applications. Prog Polym Sci. 2006;31:603-632.

16. Rinaudeo M. Main properties and current application of some polysaccharides as biomaterials. Polym Int. 2008;57(3):397-430.

17. Alonso JM, Goycoolea FM. Chitosan-polysaccharide blended nanoparticles for controlled drug delivery. In: Reis RL, editor. Natural-based Polymers for Biomedical Application. Cambridge, England: Woodhead, Ltd; 2008:644-679.

18. Pittner F. Immobilized Biomolecules in Bioanalysis. In: Schalkhammer TGM, editor. Analytical Biotechnolog. Basel, Switzerland: Birkhäuser Verlag; 2002:1-42.

19. Hoare D, Koshland D. A procedure for the selective modification of carboxyl groups in proteins. J Am Chem Soc. 1966;88:2057.

20. Al-Dubai H, Oberhofer G, Kerleta V, Hinterwirth HH, Strobl M, Gabor F. Cleaving of antibody using dihydrolipoamide and anchoring of antibody fragment onto biocompatibly coated carrier. Chemical Monthly. 2010;141:485-490.

21. Hashida S, Ishikawa E. Use of normal IgG and its fragments to lower the nonspecific binding of Fab'-enzyme conjugates in sandwich enzyme immunoassay. Anal Lett. 1985;18(B9):1143-1155.
Nanotechnology, Science and Applications

\section{Publish your work in this journal}

Nanotechnology, Science and Applications is an international, peerreviewed, open access journal that focuses on the science of nanotechnology in a wide range of industrial and academic applications. It is characterized by the rapid reporting across all sectors, including engineering, optics, bio-medicine, cosmetics, textiles, resource sustainability

\section{Dovepress}

and science. Applied research into nano-materials, particles, nanostructures and fabrication, diagnostics and analytics, drug delivery and toxicology constitute the primary direction of the journal. The manuscript management system is completely online and includes a very quick and fair peer-review system, which is all easy to use. 